\title{
Targeting epithelial-mesenchymal transition pathway in hepatocellular carcinoma
}

\author{
Jaewhan Song \\ Department of Biochemistry, Yonsei University College of Life Science and Biotechnology, Seoul, Korea
}

Keywords: Epithelial-mesenchymal transition; Phosphoinositide 3-kinase; Snails

See Article on Page 529

In this issue of Clinical and Molecular Hepatology, Lee and colleagues' publish an article entitled inhibition of phosphoinositide 3-kinase (PI3K)/protein kinase B (AKT) signaling suppresses epithelial-mesenchymal transition (EMT) in hepatocellular carcinoma (HCC) through the Snail/glycogen synthase kinase 3/betacatenin pathway, which describes a therapeutic approach to suppress the development of HCC employing PI3K inhibitors, which specifically target EMT. EMT is a highly dynamic process that occurs during normal embryonic development and cancer metastasis. ${ }^{2,3}$ It requires key drivers for mesenchymal transition, which include EMTactivating transcription factors (EMT-TFs), such as members of the Snail, Twist, and Zeb families. While EMT-TFs are known to participate in cancer metastasis, they also play roles in cancer initiation and chemoresistance, because of which they are associated with poor clinical outcomes. ${ }^{4}$ The pleiotropic roles of EMT-TFs in tumorigenesis result from their association with multiple cancer signaling pathways, including Wnt/ $\beta$-catenin, growth factor, transforming growth factor- $\beta$, Notch, inflammation, and hypoxia pathways, which can lead to the stimulation of these transcription factors and partial EMT of cancer cells. As a result of the induction of one or a combination of the abovementioned pathways, the hallmarks of EMT are exposed, which can be detected by protein markers. ${ }^{5}$ For example, there is a decreased expression of epithelial cadherin (E-cadherin), which is involved in mediating cellcell contact. This process is accompanied by the expression of vimentin, $\mathrm{N}$-cadherin, and fibronectin, which are major mesenchymal markers.

HCC is ranked the fourth most common cause of cancer-related death worldwide. ${ }^{6}$ While local ablation, surgical resection, and liver transplantation can be applied for curative treatment of HCC in the early stages, kinase and immune checkpoint inhibitors have been applied for treatment at intermediate and advanced stages. In particular, because of the well-known association of the progression of EMT with liver fibrosis, cancer stemness, and invasion, drugs targeting the EMT pathways could be employed for therapeutic applications as well. ${ }^{8}$ In line with this notion, Lee and colleagues employed PI3K inhibitors, idelalisib and LY294002, to suppress the progression of EMT in HCC.

The rationale for using PI3K inhibitors lies in the induction of EMT-TFs by well-known signaling pathways. The binding of Wnt to Frizzled induces the suppression of glycogen synthase kinase

\author{
Abbreviations: \\ AKT, protein kinase B; E-cadherin, epithelial cadherin; EGFR, epidermal growth \\ factor receptor; EMT-TFs, epithelial-mesenchymal transition-activating \\ transcription factors; EMT, epithelial-mesenchymal transition; GSK3 $\beta$, glycogen \\ synthase kinase $3 \beta ;$ HCC, hepatocellular carcinoma; PI3K, phosphoinositide \\ 3-kinase
}

\section{Corresponding author : Jaewhan Song}

Department of Biochemistry, Yonsei University College of Life Science and Biotechnology, 50 Yonsei-ro, Seodaemun-gu, Seoul 03722, Korea

Tel: +82-2-2123-5695, Fax: +82-2-362-9897

E-mail: JS0678@yonsei.ac.kr

https://orcid.org/0000-0001-8152-9210 
$3 \beta$ (GSK3 $\beta$ ) through the formation of complexes with APC, Axin, Dvl and other proteins. This leads to stabilization of $\beta$-catenin, and thereby, stimulates EMT-TFs (Fig. 1)., ${ }^{9,10}$ Inhibition of GSK3 $\beta$ could also be accomplished by activation of the epidermal growth factor receptor (EGFR)/PI3K/AKT pathway. The recruitment of growth factors to their receptors activates the cell growth pathway involving PI3K and AKT. AKT, in turn, phosphorylates GSK3 $\beta$, which is blocked from destabilizing Snail. The stabilized Snail enters the nucleus prompting the expression of factors-related to EMT (Fig. 1). ${ }^{11}$ Lee and colleagues ${ }^{1}$ employed idelalisib because this drug was approved by the Food and Drug Administration in 2013 to treat different types of leukemia. In recent years, this drug has been clinically tested for solid tumors, such as metastatic pancreatic ductal adenocarcinoma and lung cancer. ${ }^{12,13}$ Idelalisib is an ATP-competitive kinase inhibitor that targets the PI3K p110 isoform $\delta$ with high selectivity. In addition to idelalisib, Lee and colleagues $^{1}$ employed LY294002, which is a well-known chemical inhibitor of PI3K.

Treatment of HCC cell lines, Huh-BAT and HepG2, with idelalisib or LY294002 resulted in distinct changes in the morphology of cells from the epithelial to the mesenchymal state and retardation of cell growth. Furthermore, cell cycle was arrested at $G_{0} / G_{1}$ phase, with concomitant decrease in the levels of cyclin $D, E$, and cyclin-dependent kinase 6 . Transwell assays showed that treatment with idelalisib or LY294002 suppressed cell invasion and expression of E-cadherin, a hallmark of EMT. Mechanistically, both

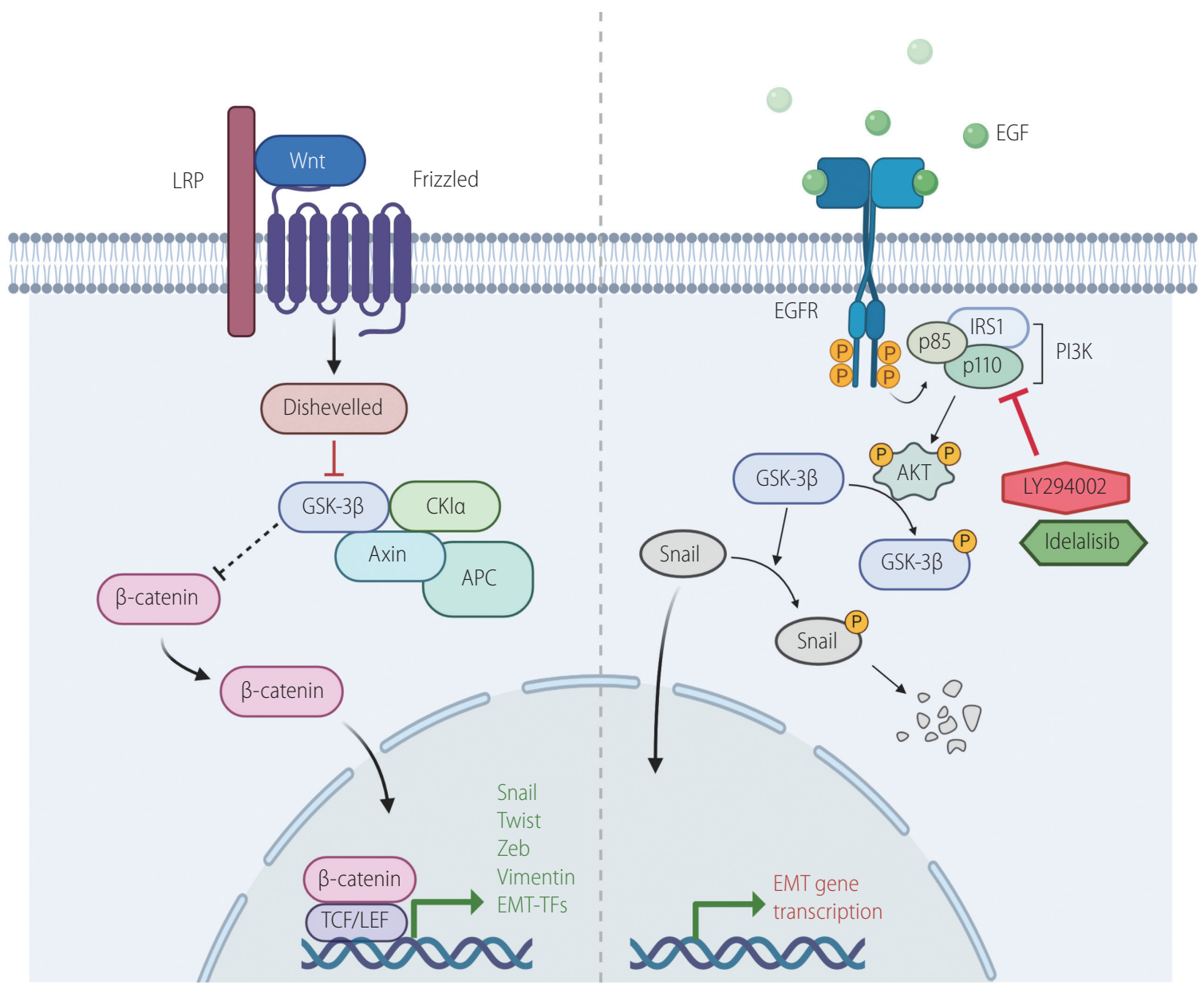

Figure 1. Regulation of EMT transcription factors by Wnt/GSK3 $\beta / \beta$-catenin/Snail and PI3K/AKT/GSK3 $/$ Snail signaling. Activation of Wnt/ $\beta$-catenin and PI3K/AKT pathways stimulates EMT pathway by suppressing GSK3 3 , which in turn is inhibited from destabilizing Snail. Suppression of GSK3 $\beta$ also stabilizes $\beta$-catenin, which in turn involved in the expression of EMT genes such as vimentin and EMT-TFs. Idelalisib and LY294002 are PI3K inhibitors, which prevent AKT activation. This leads to the release of GSK3 $\beta$ from PI3K/AKT-mediated suppression. The active GSK3 $\beta$ then phosphorylates Snail, leading to its degradation. LRP, low-density-lipoprotein-related protein; GSK3 $\beta$, glycogen synthase kinase 3ß; TCF, T-cell factor; LEF, lymphoid enhancing factor; EMT-TFs, epithelial-mesenchymal transition-activating transcription factors; EGF, epidermal growth factor; EGFR, epidermal growth factor receptor; IRS1, insulin receptor substrate 1; PI3K, phosphoinositide 3-kinase; EMT, epithelial-to-mesenchymal transition. 
the drugs induced destabilization of Snail, and caused an increase in the levels of dephosphorylated GSK3 $\beta$ in HCC cell lines, possibly due to the inhibition of the EGFR/PI3K/AKT pathway. These results were supported by the observations that GSK3 $\beta$ localized to the nucleus with the decrease in the levels of Snail and $\beta$-catenin. While this study unambiguously reveals the mechanism of action of idelalisib and LY294002, the importance of translocation of GSK3B to the nucleus upon treatment is not clearly defined, warranting further studies. Although the PI3K/AKT pathway is known to be a major pathway affecting GSK3B, the inhibitory effects of other pathways on GSK3 $\beta$ would also be interesting to investigate.

The application of idelalisib or LY294002 for treatment of HCC is important in several aspects. First, there is an aberrant activation of PI3K/AKT in HCC, which makes these signaling molecules meaningful targets. ${ }^{15,16}$ Second, GSK3 $\beta$ has been reported to play critical roles in the development of HCC. ${ }^{17}$ In particular, recent studies have indicated that GSK3 3 is associated with various signaling pathways, including Wnt, Notch, and Hedgehog, in addition to the PI3K/AKT pathway. ${ }^{17}$ As an extension to these studies, it would be interesting and necessary to target GSK3 $\beta$ with combinations of inhibitors of the related pathways.

It would be eventually required to perform studies employing genetically modified mice or orthotopic xenograft models for assessing the effects of treatment with various inhibitors, such as idelalisib and LY294002. In the former case, conditional knockout of GSK3 $\beta$ or other EMT-TFs, including Snail, in HCC mouse model would provide more definitive answers to the necessity of EMT in the development of HCC. For the latter case, it would be interesting to question whether idelalisib or LY294002 could work on an orthotopically xenografted cell line with aberrant activation of PI3K/AKT pathways. Overall, this manuscript provides an interesting perspective on the correlation between the development of HCC and EMT and offers a plausible alternative treatment for advanced HCC in future.

\section{Conflicts of Interest}

The author has no conflicts of interests to disclose.

\section{REFERENCES}

1. Lee S, Choi EJ, Cho EJ, Lee YB, Lee JH, Yu SJ, et al. Inhibition of $\mathrm{PI}$ IK/Akt signaling suppresses epithelial-to-mesenchymal transition in hepatocellular carcinoma through the Snail/GSK-3/beta-catenin pathway. Clin Mol Hepatol 2020;26:529-539.

2. Hay ED. An overview of epithelio-mesenchymal transformation. Acta Anat (Basel) 1995;154:8-20.

3. Nieto MA, Huang RY, Jackson RA, Thiery JP. EMT: 2016. Cell 2016;166:21-45.

4. Puisieux A, Brabletz T, Caramel J. Oncogenic roles of EMT-inducing transcription factors. Nat Cell Biol 2014;16:488-494.

5. Loh CY, Chai JY, Tang TF, Wong WF, Sethi G, Shanmugam MK, et al. The E-cadherin and $\mathrm{N}$-cadherin switch in epithelial-to-mesenchymal transition: signaling, therapeutic implications, and challenges. Cells 2019;8:1118.

6. Torre LA, Bray F, Siegel RL, Ferlay J, Lortet-Tieulent J, Jemal A. Global cancer statistics, 2012. CA Cancer J Clin 2015;65:87-108.

7. Vogel A, Saborowski A. Current strategies for the treatment of intermediate and advanced hepatocellular carcinoma. Cancer Treat Rev 2020;82:101946

8. Giannelli G, Koudelkova P, Dituri F, Mikulits W. Role of epithelial to mesenchymal transition in hepatocellular carcinoma. J Hepatol 2016;65:798-808.

9. Jung YS, Park JI. Wht signaling in cancer: therapeutic targeting of Wnt signaling beyond $\beta$-catenin and the destruction complex. Exp Mol Med 2020;52:183-191

10. Martin-Orozco E, Sanchez-Fernandez A, Ortiz-Parra I, Ayala-San Nicolas M. WNT signaling in tumors: the way to evade drugs and immunity. Front Immunol 2019;10:2854.

11. Manning BD, Toker A. AKT/PKB signaling: navigating the network. Cell 2017;169:381-405.

12. Burger JA, Okkenhaug K. Haematological cancer: idelalisib-targeting $\mathrm{PI} 3 \mathrm{~K} \delta$ in patients with B-cell malignancies. Nat Rev Clin Oncol 2014;11:184-186.

13. Yang Q, Modi P, Newcomb T, Quéva C, Gandhi V. Idelalisib: first-inclass PI3K delta inhibitor for the treatment of chronic lymphocytic leukemia, small lymphocytic leukemia, and follicular lymphoma. Clin Cancer Res 2015;21:1537-1542.

14. Cheah CY, Fowler NH. Idelalisib in the management of lymphoma. Blood 2016;128:331-336.

15. Grille SJ, Bellacosa A, Upson J, Klein-Szanto AJ, van Roy F, LeeKwon W, et al. The protein kinase Akt induces epithelial mesenchymal transition and promotes enhanced motility and invasiveness of squamous cell carcinoma lines. Cancer Res 2003;63:2172-2178.

16. Larue L, Bellacosa A. Epithelial-mesenchymal transition in development and cancer: role of phosphatidylinositol 3' kinase/AKT pathways. Oncogene 2005;24:7443-7454.

17. Cervello M, Augello G, Cusimano A, Emma MR, Balasus D, Azzolina $A$, et al. Pivotal roles of glycogen synthase-3 in hepatocellular carcinoma. Adv Biol Regul 2017;65:59-76. 